\title{
Vitamin B12-unresponsive methylmalonic acidemia type mut0
}

INSERM

\section{Source}

INSERM. (1999). Orphanet: an online rare disease and orphan drug data base. Vitamin B12-unresponsive methylmalonic acidemia type muto. ORPHA:289916

Vitamin B12-unresponsive methylmalonic acidemia type mut0 is an inborn error of metabolism characterized by recurrent ketoacidotic comas or transient vomiting, dehydration, hypotonia and intellectual deficit, which does not respond to administration of vitamin B12. 